\title{
CARACTERIZACIÓN DE VARIABLES METEOROLÓGICAS A SER USADAS COMO FUENTES DE ENERGÍA EN LA REGIÓN SUR DEL ECUADOR.
}

\author{
CHARACTERIZATION OF METEOROLOGICAL VARIABLES TO BE USED AS \\ SOURCES OF ENERGY IN THE SOUTHERN REGION OF ECUADOR.
}

\section{MARCO ARMIJOS-CABRERA ${ }^{1}$, VÍCTOR GONZÁLEZ-JARAMILLO ${ }^{2}$, ANDREAS FRIES ${ }^{2}$}

ESCUELA DE INGENIERÍA CIVIL ${ }^{1}$, DEPARTAMENTO DE GEOLOGÍA Y MINAS E INGENIERÍA CIVIL $^{2}$. UNIVERSIDAD TÉCNICA PARTICULAR DE LOJA. San Cayetano Alto, Marcelino Champagnat s/n, 1104608. Loja - Ecuador. mvarmijos3@gmail.com, vhgonzalez@utpl.edu.ec, aefries@utpl.edu.ec

Recibido: 13 de agosto 2018 /Aceptado: 11 de noviembre 2018

\section{RESUMEN}

El avance tecnológico a nivel mundial, conlleva al desarrollo de las sociedades, incrementando la demanda de energía eléctrica en los hogares. La energía producida actualmente es obtenida principalmente de combustibles fósiles, los cuales, aparte de no ser sustentables, contaminan el medio ambiente e incrementan la emisión de gases de efecto invernadero. En este trabajo, se presenta una alternativa al uso de combustibles fósiles para la generación de energía para consumo doméstico, específicamente la energía solar. La información necesaria respecto a la radiación solar fue derivada de imágenes satelitales (NOAAAVHRR), generando mapas promedios mensuales para evaluar el potencial de aprovechamiento de esta energía renovable en la parte sur del Ecuador y para determinar zonas posibles para la implementación de sistemas de abastecimiento. Los resultados indicaron que el aprovechamiento de energía solar es viable en los valles interandinos y las regiones sur - occidentales donde la radiación solar es fuerte y constante durante la mayoría de los días del año. Los meses con radiación solar más altos son septiembre y octubre alcanzando promedios diarios hasta $500 \mathrm{~W} / \mathrm{m}^{2}$. Estos resultados confirman que la radiación solar es una fuente potencial para la generación de energías limpias/renovables en el sur del Ecuador.

Palabras clave: Energías alternativas, Variables meteorológicas, Radiación solar, NOAA-AVHRR.

\begin{abstract}
The technological advance worldwide leads to the development of societies, increasing their energy demand. Nowadays, the energy is mainly produced by means of fossil fuels, which are not sustainable, pollute the environment and increase greenhouse gas emissions. This research presents an alternative for the generation of energy for domestic consumption without fossil fuels, specifically solar energy. The required information respective to solar radiation was derived from satellite images (NOAA-AVHRR), which were used to generate mean monthly maps to evaluate the potential of this renewable energy in southern Ecuador, and to determine possible areas for the implementation of supply systems. The results indicated that solar energy is viable in the inter- Andean valleys and the south - western regions where solar radiation is strong and constant throughout the year. The months with highest solar radiation are September and October reaching daily average values up to $500 \mathrm{~W} / \mathrm{m}^{2}$. These results confirm that solar radiation is possible sources for the generation of clean / renewable energies in southern Ecuador.
\end{abstract}

Keywords: Alternative energies, Meteorological variables, Solar radiation, NOAA-AVHRR. 


\section{INTRODUCCIÓN}

El crecimiento de las sociedades, con miras al desarrollo social y económico, así como las mejoras en la calidad de vida, salud y bienestar de las personas tiene como consecuencia una demanda elevada de energía (IPCC, 2010). La fuente primaria actualmente para la generación de energía son los combustibles fósiles (Mohtasham, 2015), como son el carbón o derivados del petróleo. El consumo de estos materiales ha causado daños visibles en el medio ambiente en varias formas (Nada, 2014), teniendo deforestación, ecosistemas marítimos y terrestres contaminados, como también emisión de gases de efecto invernadero. De ahí nace la importancia de presentar alternativas y promover tecnologías para el aprovechamiento de energías limpias como fuentes sustentables y renovables para la generación de energía eléctrica (IPCC, 2010). Una alternativa es la radiación solar, que aprovecha la energía o el calor emitido del Sol (Davis, 1990), que no contamina el ambiente ni produce gases invernaderos. La energía solar recibida en la superficie terrestre es equivale a 178000 TW/año. Sin embargo, cerca del $30 \%$ de esta energía es reflejada directamente al espacio (albedo planetario); solo el 50\% es absorbida por la superficie terrestre, el resto queda en la atmosfera (CIE, 2008). En general, la radiación solar recibida en la superficie terrestre depende principalmente de la posición y altura del Sol, como también de la nubosidad del sitio (Strahler, 2013). Durante temporadas con posición vertical del Sol, mayor energía esta recibida en la superficie, porque el camino a través de la atmosfera es más corto y menos energía esta absorbida por partículas (aerosoles) o gases. Lo mismo es válido para sitios elevados, donde el camino a través de la atmosfera es reducido, porque más energía es recibida en las cadenas montañosas. Por eso, la radiación solar muestra generalmente un aumento con la altura (gradiente positivo). Otro factor importante es la exposición y la pendiente del terreno, porque lomas orientadas al Sol reciben más energía. Sin embargo, la radiación solar depende de la nubosidad también. En sitios nublados, gran parte de la radiación solar es absorbida y reflejada por las nubes, reduciendo la energía recibida en la superficie. Debido a esto, más engería solar llega a la superficie durante épocas secas que durante épocas lluviosas, si la position del Sol es equivalente.

En el sur de Ecuador el aprovechamiento de la radiación solar es deficiente todavía, es por ello que este estudio pretende determinar zonas factibles para la implementación de estas tecnologías para promover proyectos a pequeña y mediana escala. Para determinar estas zonas se necesita cartografía adecuada, como también información meteorológica, que se puede obtener mediante imágenes satelitales o estaciones meteorológicas (Arozarena, 2002). En el sur del Ecuador, como generalmente en toda la zona tropical, la red meteorológica es escaza (González y Bosque, 2008; Fries et al., 2014), por lo que este estudio combina Sistemas de Información Geográfica (SIG) y Teledetección para localizar sitios factibles para la implementación de tecnologías sustentables para la generación de energía eléctrica.

\section{MATERIALES Y MÉTODOS}

\section{AREA DE ESTUDIO}

El área de estudio es el sur del Ecuador, e incluye las provincias El Oro, Loja y Zamora Chinchipe $\left(\sim 3^{\circ} \mathrm{S}\right.$ a $5^{\circ} \mathrm{S} ; \sim 77^{\circ} \mathrm{O}$ a $\left.81^{\circ} \mathrm{O}\right)$, las cuales cubren el territorio desde el Océano 
Pacífico hasta la Amazonia. Los rangos altitudinales varían desde el nivel del mar hasta 3800 en las partes más altas de las cadenas de los Andes. Debido a los Andes que cruzan Ecuador del norte al sur, el área de estudio es dividida en tres regiones climáticas diferentes (INAMHI, 2013; Oñate-Valdivieso et al., 2018), específicamente la Costa (oeste), la Sierra (centro) y la Amazonía (este; Figura 1).

El clima en la Costa es caracterizado por dos estaciones anuales bien marcadas (estación húmeda y estación seca). Durante el verano del hemisferio sur (diciembre hasta abril) la evaporación el más alta y consecuentemente las precipitaciones (época lluviosa), mientras que durante el invierno del hemisferio sur (junio hasta septiembre) la evaporación esta reducida (época seca) debido a la position del Sol que se encuentra vertical sobre el hemisferio norte, más lejos del área de estudio (Rollenbeck and Bendix, 2011). Las precipitaciones anuales en la Costa varían entre $500 \mathrm{~mm}$ y $2000 \mathrm{~mm}$, donde la parte sur generalmente es más seca debido a la cercanía al desierto del norte de Perú (zona de transición). Las temperaturas son más o menos constante durante todo el año $\left(\sim 26^{\circ} \mathrm{C}\right)$ en consecuencia a la ubicación del Ecuador en la zona tropical (Strahler, 2013).

El clima de la Sierra depende de la position del Sol durante el año, la cual el vertical en marzo/ abril y septiembre/ octubre, cuando se observa las precipitaciones máximas también (evaporación más alta). Al contrario de la Costa, la Sierra no muestra una estación seca bien marcada; sin embargo, el clima puede cambiar rápidamente entre los valles debido a la topografía, específicamente debido a las cadenas montañosas que forman barreras para el transporte de la humedad (Fries et al., 2014). En general, los meses más secos son agosto y septiembre, cuando la position vertical del Sol es sobre el hemisferio norte (final del invierno del hemisferio sur). Las precipitaciones como también las temperaturas dependen de la altura, alcanzando valores entre $700 \mathrm{~mm}$ y 1200 $\mathrm{mm}$ (precipitación) y $10^{\circ} \mathrm{C} \mathrm{y} 16^{\circ} \mathrm{C}$ (temperatura) donde hay centros poblados.

La Amazonia muestra un clima lluvioso durante todo el año. Sin embargo, dos máximas son observables en marzo y octubre simultáneamente con la position vertical del Sol. Las precipitaciones varían entre $2000 \mathrm{~mm}$ y $4000 \mathrm{~mm}$, dependiendo de la topografía local y la altura sobre el nivel del mar (Richter y Moreira-Muñoz, 2005). Las temperaturas son un poco más bajas comparado con la Costa (entre $20^{\circ} \mathrm{C} \mathrm{y} 22^{\circ} \mathrm{C}$ ), debida a la alta nubosidad que absorbe y refleja gran parte de la radiación solar (Fries et al., 2012).

La vegetación natural en la zona está relacionada con las regiones climáticas y sus características (Richter, 2003). En la Costa predominan sabanas, y bosques caducifolios o semi-caducifolios, mientras que en la Sierra bosques montanos y páramos (Aguirre et al., 2017; González et al., 2018). La vegetación natural en la Amazonía es bosque lluvioso tropical (Richter y Moreira-Muñoz, 2005). 


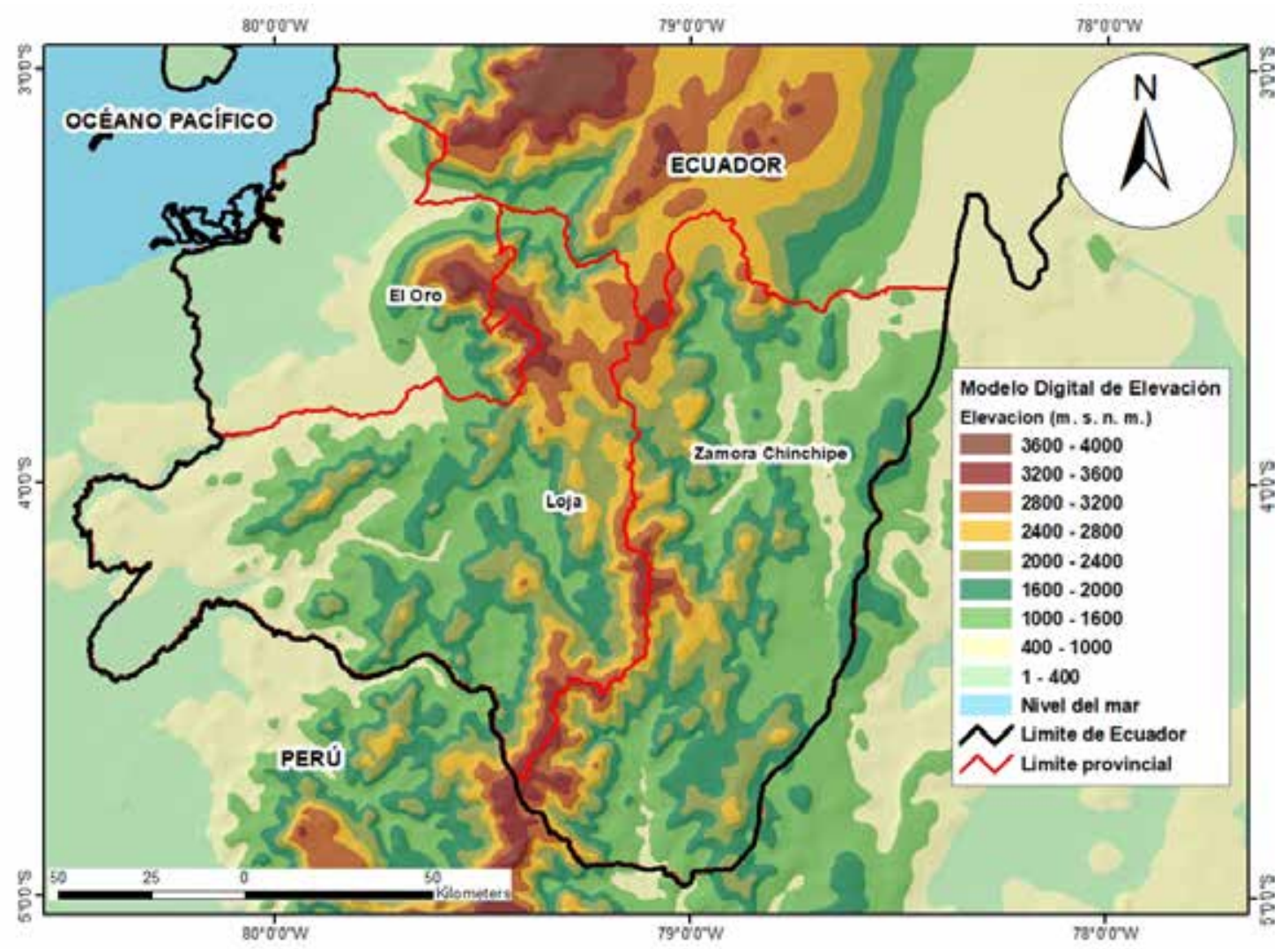

Figura 1. Área de estudio, comprende la zona sur de Ecuador.

\section{MÉTODOS}

Los mapas de radiación solar fueron generados mediante imágenes satelitales de la zona sur del Ecuador (Región 7), específicamente imágenes del satélite NOAA 17, con su sensor AVHRR, que facilita información de 5 canales espectrales en una resolución de $1.1 \mathrm{~km}$. Los canales $1(0.58 \mu \mathrm{m}-0.68 \mu \mathrm{m})$ y $2(0.725 \mu \mathrm{m}-1.10 \mu \mathrm{m})$, miden la radiación solar reflejada por la superficie en longitudes de onda de luz visible e infrarrojo cercano, respectivamente; mientras que los canales $3(3.55 \mu \mathrm{m}$ $3.93 \mu \mathrm{m}), 4(10.50 \mu \mathrm{m}-11.30 \mu \mathrm{m})$ y $5(11.50 \mu \mathrm{m}-12.50 \mu \mathrm{m})$ detectan la radiación emitida en infrarrojo medio hasta infrarrojo térmico (González-Jaramillo et al., 2016). La información utilizada consistió de 55 imágenes satelitales de los meses marzo, abril, septiembre y octubre del año 2008. Para la estimación de la radiación solar se usa las bandas 1 y 2 , que proporcionan la cantidad de energía reflejada en la superficie terrestre, la cual esta expresada en el coeficiente de reflexión o albedo de la superficie. Los canales 4 y 5 permiten estimar la radiación, proveniente del Sol. La combinación entre las dos variables (albedo y radiación solar) indica la cantidad de energía aprovechable para la generación de energía renovable. (EOI, 2012). 
Para la generación de mapas de radiación solar se utilizó el software IDRISI (versión Taiga, IDRISI, 2018), la cual permite el pre-procesamiento necesario de las imágenes satelitales respecto a errores atmosféricos y geométricos, aplicando coeficientes de calibración (NOAA, 2015). Mediante las imágenes corregidas, se calculó el albedo (bandas 1 y 2), lo cual es expresado en porcentaje con valores entre 0 y 1 (Ke), donde el valor 1 indica una reflexión completa y el valor 0 una absorción completa de la radiación solar en la superficie. Las bandas 4 y 5 presentan valores digitales entre 0 y 1023 los cuales deben ser ajustados mediante ecuaciones dadas por NOAA (2015), que convierten los dígitos en temperaturas absolutas $(\mathrm{T})$ en grados Kelvin $\left({ }^{\circ} \mathrm{K}\right)$. Para estimar la energía solar en $\mathrm{W} / \mathrm{m}^{2}$ y calcular la energía solar aprovechable dentro de cada celda $(\mathrm{x}, \mathrm{y})$, se aplicó la ecuación de Stefan- Boltzmann multiplicando el albedo (Ecuación 1; Vásquez, 2014):

$$
\mathrm{H}=\mathrm{Ke}^{*} \sigma^{*} \mathrm{~T}^{4}
$$

Dónde:

$\mathrm{H}=$ Energía solar sobre la superficie terrestre expresado en $\left(\mathrm{W} / \mathrm{m}^{2}\right)$

$\mathrm{Ke}=$ Constante de emisividad dependiendo del cuerpo entre 0 y 1 (albedo).

$\sigma=$ La constante de Stefan - Boltzmann $\left(5,6703+10^{-8}\right)$ expresado en $\left(\mathrm{W} / \mathrm{m}^{2} * \mathrm{~K}^{4}\right)$

$\mathrm{T}^{4}=$ Temperatura absoluta $\left({ }^{\circ} \mathrm{K}\right)$

Sin embargo, esta metodología solo es aplicable para celdas que no presentan nubes, porque la nubosidad impide el análisis de la superficie terrestre (González-Jaramillo et al., 2016). La discriminación de píxeles nublados como también del tipo de superficie se realizó mediante la metodología descrita por Bendix et al. (2004).

\section{RESULTADOS Y DISCUSIÓN}

Los mapas de radiación solar fueron generados para los meses extremos, específicamente marzo/ abril (final de la estación lluviosa) y septiembre/ octubre (final de la estación seca; INAMHI, 2013). La Figura 2 presenta el mapa para el mes de marzo de la radiación aprovechable para la generación de energía solar (Ecuación 1; Vásquez, 2014), indicando una radiación promedia entre de 200 a $400 \mathrm{~W} / \mathrm{m}^{2}$, lo que concuerda con el balance energético según CIIFEN (2000). Los valores más altos se observan en la Costa (provincia El Oro) como también en la parte central y sur-occidental de la provincia de Loja donde la nubosidad es generalmente baja. Para la Amazonia valores mínimos fueron calculados debido a la nubosidad frecuente sobre esta región (Bendix et al., 2009). Las cadenas de los Andes muestran valores bajos también, especialmente en las cumbres, donde la alta nubosidad reduce la radiación solar (Fries et al., 2012). 

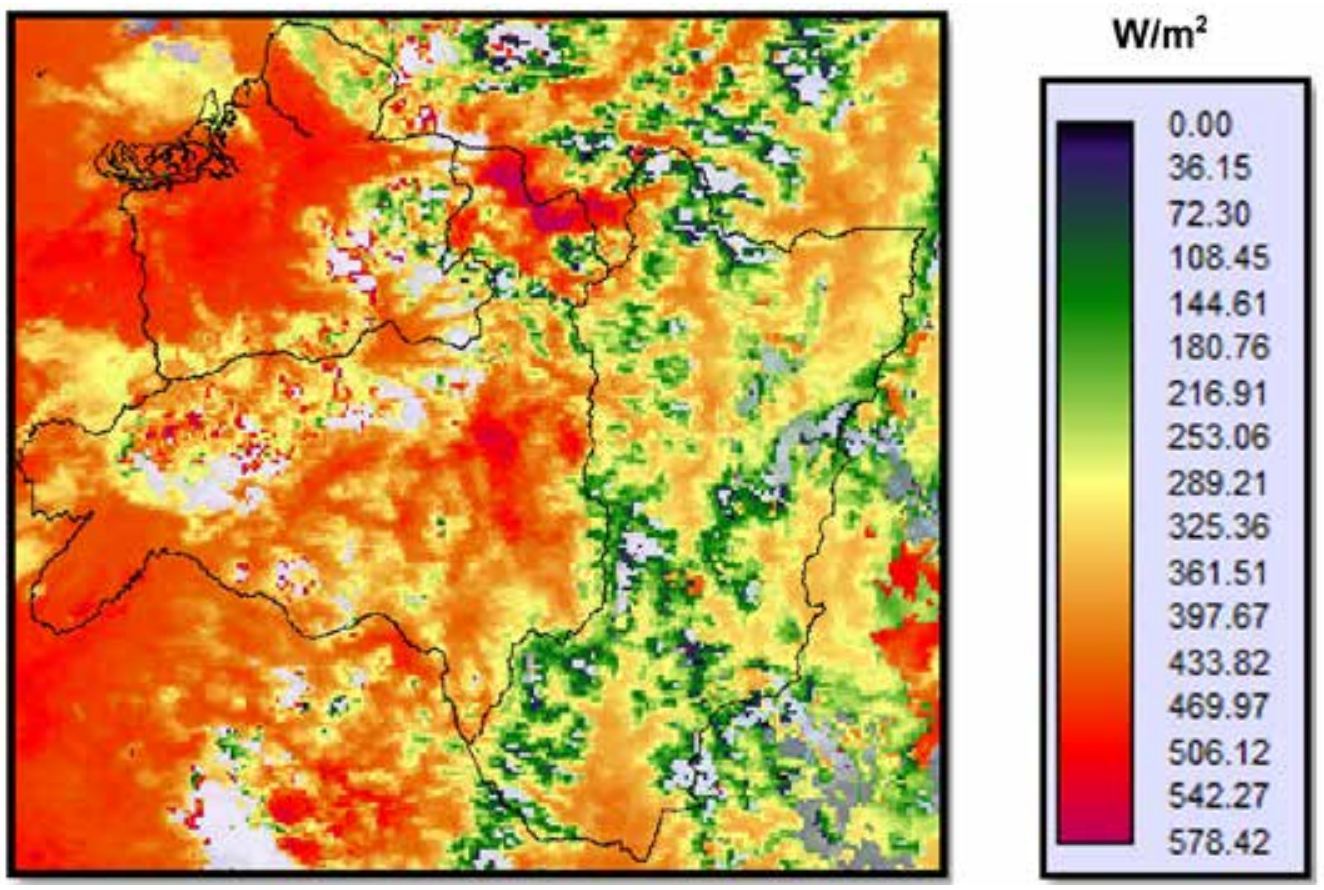

Figura 2. Radiación solar promedio en el mes de marzo de 2008.

La radiación solar en el mes de abril (Fig. 3) muestra la misma distribución que el mes de marzo (Fig. 2), aunque los valores calculados son un poco más bajos. Esto se debe la formación frecuente de tormentas durante la estación lluviosa en el sur del Ecuador (Fries et al., 2014), la cual reduce la radiación recibida en la superficie. Las tormentas se forman generalmente alrededor de media día y en las tardes cuando la radiación solar es más fuerte también (10 am a las 03 pm; Orellana y Sarango, 2015). En la Figura 3 los sistemas grandes de nubes se observan sobre la Amazonia y sobre la Costa, como también sobre las cadenas montañosas, donde la radiación solar es particularmente baja. Sin embargo, la parte central y sur-occidental de la provincia de Loja muestra valores altos, que indica que la nubosidad sobre estas regiones es menos frecuente.

Durante la estación seca (septiembre y octubre) la nubosidad es generalmente baja en la zona de estudio, específicamente en la Costa y sobre los valles interandinos, porque la radiación solar es más intensa alcanzando valores máximos promedios sobre $500 \mathrm{~W} /$ $\mathrm{m}^{2}$ (CIIFEN, 2000). Sin embargo, neblinas son frecuentes en la franja costera, lo cual reduce la radiación recibida en la superficie. La Figura 4 indica la radiación solar durante el mes de septiembre, donde se observa valores muy altos, específicamente la provincia de Loja y partes de la Costa (El Oro) no cubiertos de neblina. Los valores máximos se encuentran en los valles interandinos y en la parte sur-occidental de la provincia de Loja (zona de transición al desierto del norte de Perú), donde nubosidad generalmente es ausente durante esta época del año (Rollenbeck and Bendix, 2011; Bendix et al., 2017). Volares mínimos ocurren sobre la Amazonia y en las cadenas montañosas, debido a la alta nubosidad, como también en la franja costera, donde neblinas son frecuentes. 

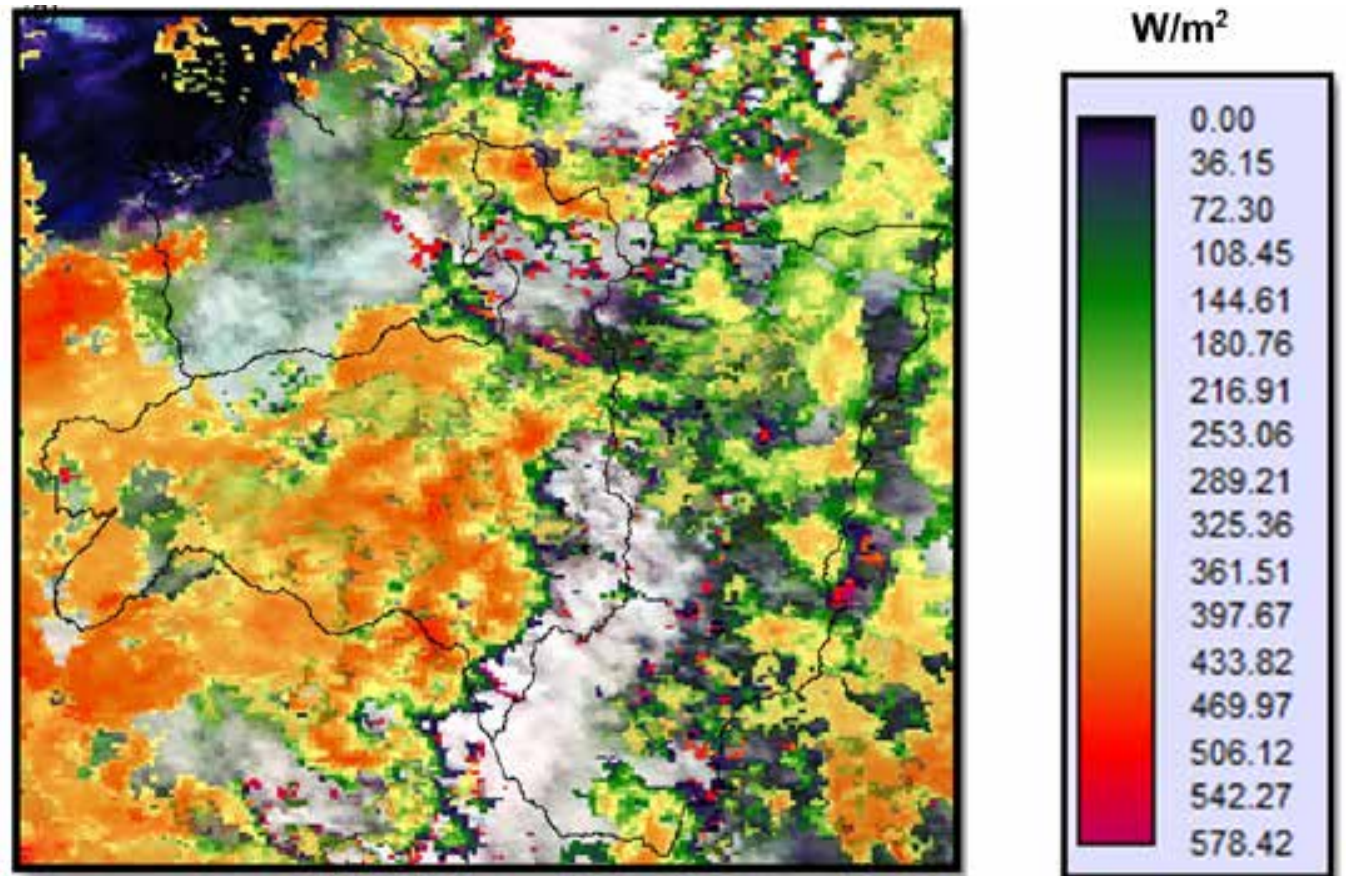

Figura 3. Radiación solar promedio en el mes de abril de 2008.
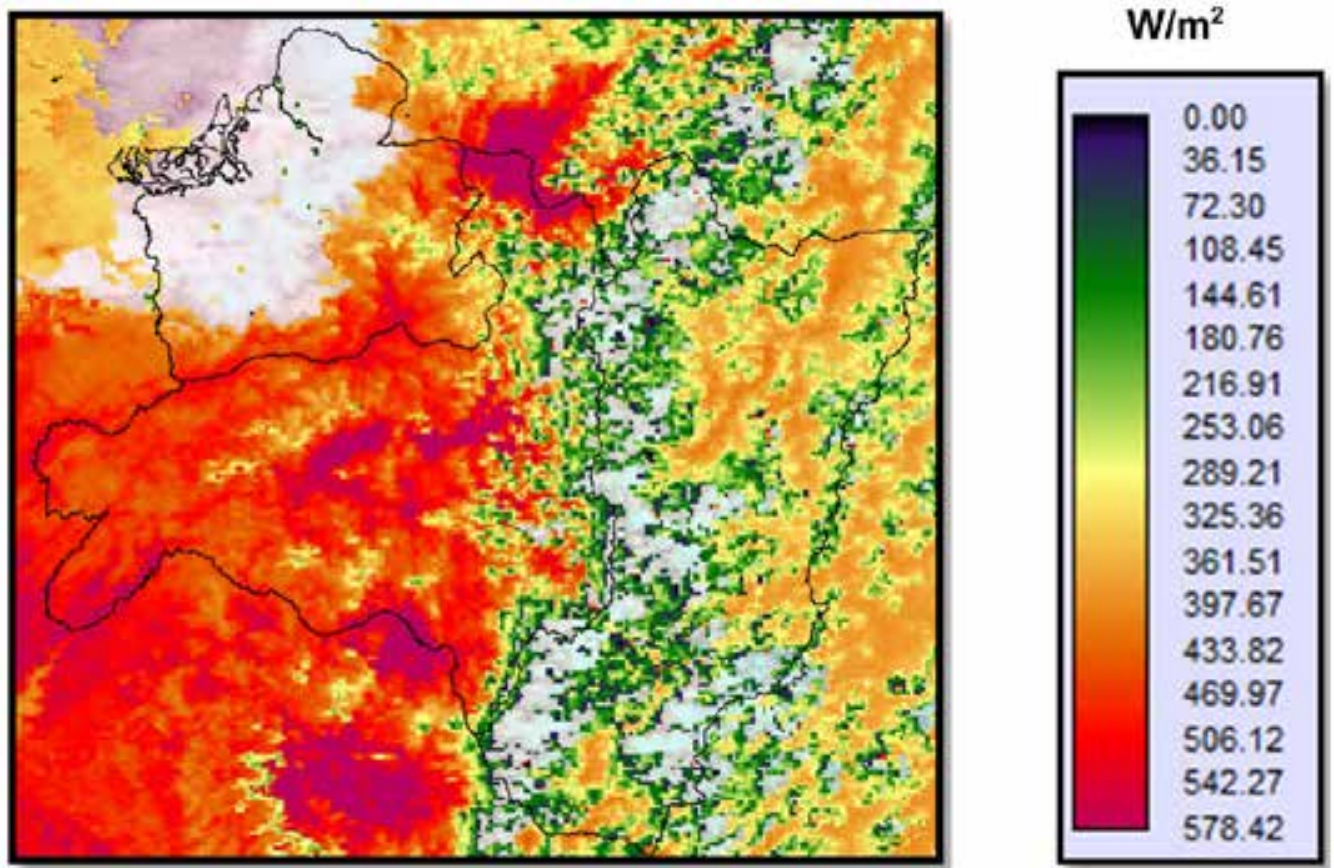

Figura 4. Radiación solar promedio en el mes de septiembre de 2008. 
La Figura 5 muestra la radiación solar en octubre, la cual indica la misma distribución que en septiembre (Fig.4). Sin embargo, los valores en la Amazonia y en la parte oriental de la provincia de Loja son más bajos, porque octubre es el mes de transición entre la estación seca y la estación húmeda, y la formación de tormentas comienza (Rollenbeck and Bendix, 2011). El inicio de la estación lluviosa es visible sobre la Costa también, donde algunas áreas muestran valores muy bajos, debido a las nubes de tormenta que absorben y reflejan gran cantidad de la radiación incidente. Los valores máximos se observan sobre los valles interandinos (provincia de Loja) como en la parte sur-occidental del área de estudio. La radiación extremadamente alta sobre los valles interandinas se debe a la posición vertical del Sol sobre el área de estudio y las cadenas montañosas que impiden el transporte de la humedad (barreras; Fries et al., 2014; Oñate-Valivieso et al., 2018).

En resumen, la radiación solar en el sur de Ecuador es generalmente alta durante todo el año, debido a la su ubicación en la zona tropical (Strahler, 2013). Solo en regiones donde nubosidad es frecuente (Amazonia y cadenas montañosas) la energía recibida en la superficie es reducida (Bendix et al., 2008; González-Jaramillo et al., 2016). Las regiones más favorables para la implementación de sistema de abastamiento para la generación de energía son los valles interandinos como también la parte sur-occidental del área de estudio (transición al desierto), donde la nubosidad es generalmente baja (Bendix et al., 2017). Los valores calculados (entre $200 \mathrm{~W} / \mathrm{m}^{2}$ a $600 \mathrm{~W} / \mathrm{m}^{2}$ ) coinciden con los resultados de estudios previos (CIIFEN, 2000; Orellana y Sarango 2015), indicando que la radiación solar es una fuente potencial para la generación de energías limpias/renovables en el sur del Ecuador.
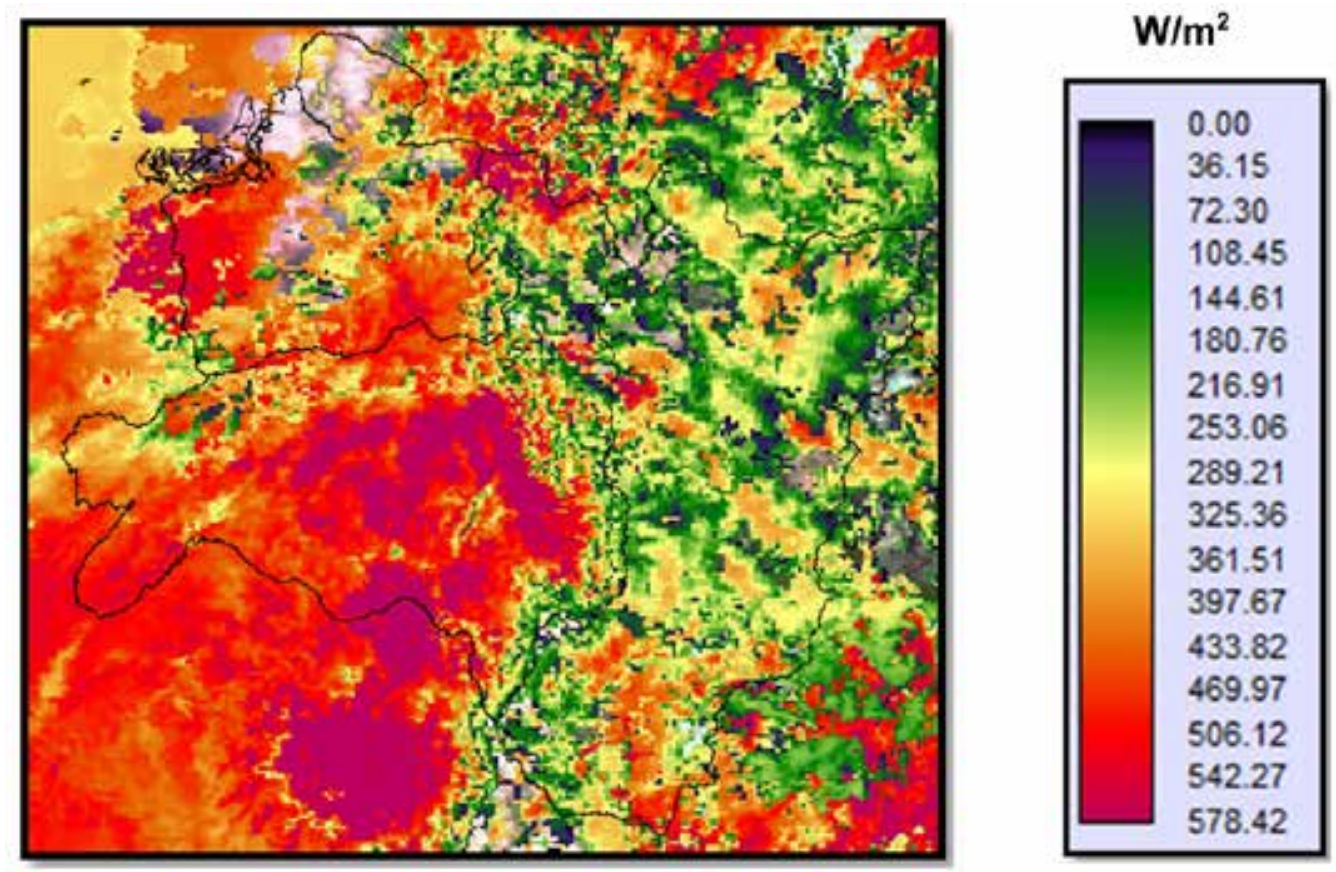

Figura 5. Radiación solar promedio en el mes de octubre de 2008. 


\section{CONCLUSIONES}

Este estudio muestra que la estimación de la radiación solar aprovechable para la generación de energía es factible mediante SIG y Teledetección para regiones en donde estaciones meteorológicas son escasas o inexistentes. Las diferentes bandas de los satélites (p.ej. NOAA-AVHRR) permiten el cálculo realista de la radiación solar en alta resolución con un error máximo estimado de $5 \%$ en sitios con cielos despejados y $15 \%$ para cielos nublados (Vera, 2005). En base de los mapas generados se puede identificar el potencial en las diferentes regiones para la implementación de sistemas de abastamiento de este tipo de energía renovable.

En general, Ecuador es favorecido respecto a la radiación solar debido a su posición geográfica en la zona tropical donde la energía solar es alta hasta extrema durante todo el año (WHO, 2003). Respecto a la parte sur del Ecuador (Región 7), los sitios más favorables para la implementación de sistemas para la generación de energía solar son los valles interandinos y la parte sur-occidental (zona de transición al desierto), donde la nubosidad es generalmente baja. La Amazonia, como también las cadenas montañosas son frecuentemente cubiertas de nubes por lo que la generación de energía solar es limitada. Sin embargo, las cumbres pueden proveer otra fuente de energía renovable (EKOS, 2014), específicamente energía eólica, debido a los vientos fuertes y constantes observados en estos sitios (Windhorst et al., 2013).

Estos resultados pueden servir como una guía referencial para la implementación potencial de sistemas de generación eléctrica a pequeña y mediana escala, realizando adicionalmente un análisis de factibilidad de ejecución y de costos.

\section{REFERENCIAS}

Aguirre-Mendoza, Z., Aguirre, N., Merino, B., Ochoa, I. (2015). Los páramos del Parque Nacional Podocarpus: Una aproximación a su diversidad ecosistémica y florística. En: Cambio climático y Biodiversidad: Estudio de caso de los páramos del Parque Nacional Podocarpus; Aguirre, N., Ojeda, T., Eguiguren, E., Aguirre, Z., Eds.; Ediloja: Loja, Ecuador, 2015; pp. 22-45.

Arozarena, A. (2002). Sistemas de captura y análisis de la información territorial. Madrid España. Cap.3.

Bendix. J., Rollenbeck. R., Palacios. E.W. (2004). Cloud detection in the tropics- a suitable tool for climate- ecological studies in the high mountains of Ecuador. Faculty of Geography, University of Marburg. Marburg - Germany.

Bendix, J., Rollenbeck, R., Richter, M., Fabian, P., Emck, P. (2008). Climate. In Gradients in a Tropical Mountain Ecosystem of Ecuador; Beck, E., Bendix, J., Kottke, I., Makeschin, F., Mosandl, R., Eds.; Springer: Berlin/Heidelberg, Germany, 2008; Volume 198, pp. 63-74.

Bendix, J., Trachte, K., Cermak, J., Rollenbeck, R., Nauß, T. (2009). Formation of convective clouds at the foothills of the tropical eastern Andes (South Ecuador). J Appl Meteorol, 48, 1-17. 
Bendix, J., Fries, A., Zárate, J., Trachte, K., Rollenbeck, R., Pucha-Cofrfrep, F., . . Achilles, S. (2017). RadarNet-sur first weather radar network in tropical high mountains. Bulletin of the American Meteorological Society, 98(6), 1235-1254. doi:10.1175/BAMS-D-15-00178.1

CIE. (Corporación para la Investigación Energética). (2008). Atlas solar del Ecuador con fines de Generación Eléctrica. Quito - Ecuador. 5-7.

CIIFEN. (2000). (Centro internacional para la investigación del fenómeno del niño. Guayaquil, Ecuador. http://www.ciifen.org/index.php\%3Foption\%3Dcom_content\%26view\%3Dcate gory\%26layout\%3Dblog\%26id\%3D99\%26Itemid\%3D132\%26lang\%3Des Ingresado: 5 de mayo de 2015.

Davis, G. (1990). Energy for planet earth. Scientific American, 263, 3, 20-7.

EKOS. (2014). Central Eólica Villonaco le ahorra a Ecuador USD 13,2 millones. http://www. ekosnegocios.com/negocios/verArticuloContenido.aspx?idArt=3314. Ingresado: 6 de octubre de 2015.

EOI. 2012. Radiación directa, difusa y reflejada. http:/www.eoi.es/wiki/index. php/Radiaci\%C3\%B3n_directa,_difusa_y_reflejada_en_Ecomateriales_y_ construcci\%C3\%B3n_sostenible. Ingresado: 15 de septiembre de 2017.

Fries, A., Rollenbeck, R., Nauss, T., Peters, T., Bendix, J. (2012). Near surface air humidity in a megadiverse Andean mountain ecosystem of southern Ecuador and its regionalization. Agricultural and Forest Meteorology, 152, 17-30.

Fries, A., Rollenbeck, R., Bayer, F., Gonzalez, V., Oñate-Valivieso, F., Peters, T., Bendix, J. (2014). Catchment precipitation processes in the San Francisco valley in southern Ecuador: combined approach using high-resolution radar images and in situ observations. Meteorol. Atmos. Phys, 126, 13-29, DOI:10.1007/s00703-014-0335-3.

González. F., D., y Bosque. S., J. (2008). Generación de un mapa de vientos en un sig. Boletín de la A.G.E. $\mathrm{N}^{\circ}$ 47. Universidad de Alcalá. Madrid - España. p.51-77.

González-Jaramillo, V., Fries, A., Rollenbeck, R., Paladines, J., Oñate-Valdivieso, F., Bendix, J. (2016). Assessment of deforestation during the last decades in Ecuador using NOAAAVHRR satellite data. Erdkunde, 70, 217-235, DOI:10.3112/erdkunde.2016.03.02.

González-Jaramillo, V., Fries, A., Zeilinger, J., Homeier, J., Paladines-Benitez, J., Bendix, J. (2018). Estimation of above ground biomass in a tropical mountain forest in southern ecuador using airborne LiDAR data. Remote Sensing, 10(5) doi:10.3390/rs10050660

IDRISI. (2018). Remote Sensing Guide Individual Tutorials by Chapter. https://clarklabs.org/ download/remote-sensing-guide-tutorial-data/. Ingresado: 10 de marzo de 2017.

INAMHI (1990-2013). Anuarios Meteorológicos. Quito, Ecuador. http://www. serviciometeorologico.gob.ec/biblioteca/. Ingresado: 30 de octubre de 2017.

IPCC. (2010). Informe especial sobre fuentes de energía renovables y mitigación del cambio climático. Informe del Grupo de trabajo III del Grupo Intergubernamental de Expertos sobre el Cambio Climático. https://www.ipcc.ch/pdf/special-reports/srren/srren_report_ es.pdf. Ingresado: 3 de octubre de 2017

Mohtasham, J. (2015). Review Article-Renewable Energies. Energy Procedia, 74, 1289 - 1297.

NOAA, 2015. NOAA KLM User's Guide, Section 7.1, AVHRR. http://www.ncdc.noaa.gov/oa/ podguide $/ \mathrm{ncdc} / \mathrm{docs} / \mathrm{klm} / \mathrm{html} / \mathrm{c} 7 / \mathrm{sec} 7-1$.htm. Ingresado: 20 de febrero de 2017.

Nada, Kh. M. A. (2014). Renewable Energy Types. Journal of Clean Energy Technologies, $2,1,61-64$. 
Oñate-Valdivieso, F., Fries, A., Mendoza, K., Gonzalez-Jaramillo, V., Pucha-Cofrep, F., Rollenbeck, R., \& Bendix, J. (2018). Temporal and spatial analysis of precipitation patterns in an andean region of southern ecuador using LAWR weather radar. Meteorology and Atmospheric Physics, 130(4), 473-484. doi:10.1007/s00703-017-0535-8

Orellana, A., y Sarango. J. (2015). Estudio de factibilidad para el uso de energía solar y eólica en sistemas de alumbrado público para la vía de integración barrila, sector el Plateado servido por empresa eléctrica regional del Sur. Tesis de Ingeniería. Ecuador, Cuenca.

Richter, M. (2003): Using epiphytes and soil temperaturas for eco-climatic interpretations in southern Ecuador. Erdkunde, 57 (3), 161-181. DOI: 10.3112/erdkunde.2003.03.01

Richter, M., \& Moreira-Muñoz, A. (2005). Heterogeneidad climática y diversidad vegetacional en el sur de Ecuador: un método de fitoindicación (in Spanish). Revista Peruana de Biología, $12,217-238$.

Rollenbeck, R., y Bendix, J. (2011). Rainfall distribution in the Andes of southern Ecuador derived from blending weather radar data and meteorological field observations. Atmos Res, 99, 277-289.

Strahler, A., H. (2013). Introducing Physical Geography (6th ed.). USA: Wiley. ISBN: 978-1118-39620-9

Vera, N. (2005). “Atlas climático de irradiación solar a partir de imágenes del satélite NOAA. Aplicación a la península Ibérica, Barcelona (Tesis Doctoral de Vera Mella Nelson, Barcelona 2005).

Vázquez, C. (2014). Análisis, modelamiento y gestión de una micro red eléctrica para edificaciones residenciales usando paneles fotovoltaicos. Tesis de Ingeniería. Ecuador, Quito.

Windhorst, D., Waltz, T., Timbe, E., Fred, H. G., Breuer, L. (2013). Impact of elevation and weather patterns on the isotopic composition of precipitation in a tropical montane rainforest. Hydrol Earth Syst Sci, 17, 409-419

World Health Organization. (2003): ÍNDICE UV SOLAR MUNDIAL: GUIA PRACTICA. Catalogación por la Biblioteca de la OMS. ISBN 9241590076 